\title{
MULTIPLICATEURS ET CLASSES CARACTÉRISTIQUES
}

\author{
PAR \\ J. L. KOSZUL
}

Introduction. Soient $Y$ un espace topologique, $R$ une relation d'équivalence dans $Y$ et $Y_{1}$ le graphe de cette relation $R$. Soit d'autre part $G$ un groupe topologique. Les espaces fibrés principaux $\left({ }^{1}\right)$ de base $Y / R$, de groupe $G$, qui sont trivialisés par l'application canonique $Y \rightarrow Y / R$, c'est à dire dont l'image réciproque par cette application est un espace fibré trivial de base $Y$, peuvent se définir au moyen de certaines applications continues de $Y_{1}$ dans $G$ (les multiplicateurs). Ce mode de définition des espaces fibrés se rencontre lorsqu'on définit un espace fibré par un système de fonctions de passage, ou encore lorsque, dans la théorie des variétés complexes, on définit une classe de diviseurs par un facteur d'automorphie. Le but de cette Note est d'indiquer un procédé de formation des classes caractéristiques d'un espace fibré principal défini par la donnée d'un multiplicateur. Ce procédé repose sur une construction des groupes d'homologie de l'espace quotient $Y / R$ qui est étroitement apparentée, à la fois à la "bar-construction" de Eilenberg-MacLane, et à certains résultats de $\mathrm{H}$. Cartan et J. Leray sur les groupes discrets de transformations. Notre construction n'a cependant pas le caractère strictement algébrique des constructions envisagées par ces auteurs.

1. Homologie des espaces quotients. Dans ce paragraphe, on désignera par $Y$ un espace topologique et par $R$ une relation d'équivalence dans $Y$. Pour tout entier $r \geqq 0$, soit $Y_{r}$ le sous-espace du produit direct $Y^{r+1}$ constitué par les points $\left(y_{0}, y_{1}, \cdots, y_{r}\right)$ tels que $y_{i} \sim y_{0}$ pour $i=1,2, \cdots, r$. Pour tout couple d'entiers $(r, i)$ tels que $r>0$ et $r \geqq i \geqq 0$, on désignera par $a_{r, i}$ l'application continue de $Y_{r}$ dans $Y_{r-1}$ définie par

$$
a_{r, i}\left(y_{0}, y_{1}, \cdots y_{r}\right)=\left(y_{0}, \cdots, \hat{y}_{i}, \cdots, y_{r}\right) .
$$

Lorsque $r \geqq 2$ et $r \geqq j>i \geqq 0$, on a: $a_{r-1, j-1} a_{r, i}=a_{r-1, i} a_{r, j}$. On notera $A_{r, p}(R, Y)$, ou simplement $A_{r, p}$, le groupe des chaînes singulières de degré $p$ de l'espace $Y_{r}$, les coefficients étant entiers. Soit $A(R, Y)$ le groupe abélien bigradué, somme directe des groupes $A_{r, p}$. On définit dans $A(R, Y)$ deux opérateurs bords:

(1) l'opérateur $\partial^{\prime}$, de type $(0,-1)$, dont la restriction à $A_{r}=\sum_{p} A_{r, p}$ est $(-1)^{r} \partial, \partial$ désignant l'opérateur bord du complexe des chaînes singulières de $Y_{r}$,

(2) l'opérateur $\partial^{\prime \prime}$, de type $(-1,0)$, dont la restriction à $A_{r}$ est

Received by the editors January 31, 1957.

(1) Il ne sera question que d'espaces fibrés localement triviaux et où le groupe opère a droite. 
$\sum_{i=0}^{i=r}(-1)^{i} \alpha_{r, i}, \alpha_{r, i}$ désignant l'homomorphisme de $A_{r}$ dans $A_{r-1}$ défini par l'application $a_{r, i}\left(\partial^{\prime \prime}=0 \operatorname{sur} A_{0}\right)$.

On a visiblement les relations: $\partial^{\prime 2}=\partial^{\prime \prime 2}=\partial^{\prime} \partial^{\prime \prime}+\partial^{\prime \prime} \partial^{\prime}=0$. On définit donc un nouvel opérateur bord $\Delta$, abaissant le degré total d'une unité, en posant $\Delta=\partial^{\prime}+\partial^{\prime \prime}$. Les groupes d'homologie (respectivement, les modules de cohomologie $\left({ }^{2}\right)$ ) du complexe $A(R, Y)$ ainsi défini seront notés $H_{n}(R, Y)$ (resp. $\left.H^{n}(R, Y)\right)$.

On va démontrer que, si $R$ vérifie certaines conditions, les groupes $H_{n}(R, Y)$ et les modules $H^{n}(R, Y)$ sont respectivement isomorphes aux groupes d'homologie singulière $H_{n}(Y / R)$ et aux modules de cohomologie singulière $H^{n}(Y / R)$ de l'espace quotient $Y / R$. L'application canonique $q$ de $Y$ sur $Y / R$ définit un homomorphisme $\tilde{\chi}$ de $A_{0}$ dans le groupe $S(Y / R)$ des chaînes singulières de $Y / R$.

LEMME 1. La suite

$$
S(Y / R) \stackrel{\chi}{\leftarrow} A_{0} \stackrel{\partial^{\prime \prime}}{\leftarrow} A_{1} \stackrel{\partial^{\prime \prime}}{\leftarrow} A_{2} \ldots
$$

est une suite exacte.

Soit en effet $\tau$ un simplexe singulier de $Y / R$ et soit $A_{r}(\tau)$ le sous-groupe de $A$, engendré par les simplexes singuliers de $Y_{r}$ qui sont de la forme $\left(\tau_{0}, \tau_{1}, \cdots, \tau_{r}\right)$, où les $\tau_{i}$ sont des simplexes singuliers de $Y$ tels que $q\left(\tau_{i}\right)=\tau$. On a $\partial^{\prime \prime} A_{r}(\tau) \subset A_{r-1}(\tau)$ pour tout $r>0$. D'autre part, la suite

$$
Z_{\tau} \stackrel{\chi}{\leftarrow} A_{0}(\tau) \stackrel{\partial^{\prime \prime}}{\leftarrow} A_{1}(\tau) \stackrel{\partial^{\prime \prime}}{\leftarrow} A_{2}(\tau) \cdots
$$

est une suite exacte comme le montre l'opérateur d'homotopie $h$ défini lorsque $A_{0}(\tau) \neq(0)$ en choisissant un simplexe singulier $\tau^{\prime}$ de $Y$ tel que $q\left(\tau^{\prime}\right)=\tau$ et en posant $h\left(\tau_{0}, \tau_{1}, \cdots, \tau_{r}\right)=\left(\tau^{\prime}, \tau_{0}, \tau_{1}, \cdots, \tau_{r}\right)$. Puisque chaque groupe $A_{r}$ est somme directe des groupes $A_{r}(\tau)$, où $\tau$ parcourt l'ensemble des simplexes singuliers de $Y / R$, ceci démontre le Lemme.

On a évidemment $\chi \partial^{\prime}=\partial \chi$; ceci permet de prolonger $\chi$ en un homomorphisme du complexe $A(R, Y)$ dans le complexe $S(Y / R)$ en posant $\chi\left(A_{r}\right)$ $=(0)$ pour $r>0$.

ThÉORème 1. Soit $S^{\prime}(Y / R)$ le sous-complexe de $S(Y / R)$ image de $A(R, Y)$ par $\chi$. Si les groupes d'homologie du complexe $S(Y / R) / S^{\prime}(Y / R)$ sont tous nuls, alors $\chi$ induit, pour tout entier $n$, un isomorphisme canonique $\chi_{*}: H_{n}(R, Y)$ $\rightarrow H_{n}(Y / R)$ et un isomorphisme canonique $\chi^{*}: H^{n}(Y / R) \rightarrow H^{n}(R, Y)$.

Soit en effet $N$ le noyau de l'homomorphisme prolongé $\chi$. Le Lemme 1 montre que dans chaque classe d'homologie de $N$, il existe un cycle sans composantes dans $A_{0}+A_{1}+\cdots A_{r}$, et ceci quel que soit l'entier $r$. Comme

(2) Les modules de cohomologie d'un complexe de chaines $C_{n}$ seront formés à partir du complexe de cochaines $\operatorname{Hom}_{Z}\left(C_{n}, k\right)$, ou $k$ est un anneau commutatif choisi une fois pour toutes. 
$A_{r}$ ne contient pas d'élément $\neq 0$ de degré $<r$, ceci prouve que les groupes d'homologie du complexe $N$ sont tous nuls. On en déduit le Théorème 1 en considérant la suite exacte de complexes:

$$
(0) \leftarrow S(Y / R) / S^{\prime}(Y / R) \leftarrow S(Y / R) \stackrel{\chi}{\leftarrow} A(R, Y) \leftarrow N \leftarrow(0) .
$$

On observera que les groupes d'homologie de $S(Y / R) / S^{\prime}(Y / R)$ sonc tous nuls, notamment lorsque $Y$ est un espace fibré localement trivial de base $Y / R$, et plus généralement, lorsque tout point $x \in Y / R$ possède un voisinage $U$ tel que tout simplexe singulier dans $U$ soit image par $q$ d'un simplexe singulier de $Y$ (cf. [4, Chapter VII, §8]).

EXEmple. Soit $X$ un espace topologique et soit $\left(U_{i}\right)_{i \in I}$ un recouvrement ouvert de $X$. Dans le sous-espace $Y \subset X \times I$ constitué par les points $(x, i)$ tels que $x \in U_{i}$, soit $R$ la relation d'équivalence pour laquelle $(x, i) \sim\left(x^{\prime}, j\right)$ lorsque $x=x^{\prime}$. L'espace $X$ s'identifie au quotient $Y / R$. Un point de $Y_{r}$ est un point de $Y^{r+1}$ de la forme $\left(\left(x, i_{0}\right),\left(x, i_{1}\right), \cdots,\left(x, i_{r}\right)\right)$ où $x \in U_{i_{0}} \cap U_{i_{1}} \cap \cdots$ $\cap U_{i_{r}}$ On peut donc identifier l'espace $Y_{r}$ au sous-espace des points $\left(x, i_{0}, i_{1}, \cdots, i_{r}\right)$ de $X \times I^{r+1}$ tels que $x \in U_{i_{0}} \cap U_{i_{1}} \cap \cdots \cap U_{i_{r}}$. Posons $M_{r}=\partial^{\prime} A_{r, 1}+\sum_{p>0} A_{r, p}$ et $M=\sum_{r} M_{r}$. Le sous-groupe $M$ est un souscomplexe de $A(R, Y)$. Si toute intersection non vide d'une famille finie d'ouverts du recouvrement a même homologie singulière qu'un point, alors pour tout entier $n$, on a $H_{n}(M)=(0)$ et $H_{n}(X) \simeq H_{n}(R, Y) \simeq H_{n}(A(R, Y) / M)$ est isomorphe au $n$-ième groupe d'homologie du nerf du recouvrement. On obtient un résultat analogue en cohomologie, qui est un cas particulier du résultat bien connu de J. Leray ramenant la cohomologie d'un espace à celle du nerf d'un recouvrement simple [6].

2. Homologie et groupes de transformations. Dans ce paragraphe, on désignera par $G$ un groupe topologique et par $Y$ un espace topologique dans lequel $G$ opère de manière continue à droite. Partant de ces données, on va construire un complexe analogue au complexe $A(R, Y)$ défini au paragraphe 1. Comme on le montrera du reste, cette nouvelle construction coïncide avec la précédente lorsque $Y$ est un espace fibré principal de groupe $G$.

Pour tout couple d'entiers $(r, i)$ où $r>0$ et $r \geqq i \geqq 0$, on désignera par $b_{r, i}$ l'application continue de $Y \times G^{r}$ dans $Y \times G^{r-1}$ définie par

$$
\begin{aligned}
& b_{r, 0}\left(y, s_{1}, s_{2}, \cdots, s_{r}\right)=\left(y s_{1}, s_{2}, \cdots, s_{r}\right), \\
& b_{r, i}\left(y, s_{1}, s_{2}, \cdots, s_{r}\right)=\left(y, s_{1}, \cdots, s_{i} s_{i+1}, \cdots, s_{r}\right) \quad(0<i<r), \\
& b_{r, r}\left(y, s_{1}, s_{2}, \cdots, s_{r}\right)=\left(y, s_{1}, \cdots, s_{r-1}\right) .
\end{aligned}
$$

Lorsque $r \geqq 2$ et $r \geqq j>i \geqq 0$, on $a: b_{r-1, j-1} b_{r, i}=b_{r-1, i} b_{r, j}$. On notera $B_{r, p}(G, Y)$, ou simplement $B_{r, p}$, le groupe des chaînes singulières de degré $p$ de l'espace $Y \times G^{r}$. Soit $B(G, Y)$ le groupe abélien bigradué somme directe des groupes $B_{r, p}$. On définit dans $B(G, Y)$ deux opérateurs bord: 
(1) l'opérateur $\partial^{\prime}$, de type $(0,-1)$, dont la restriction à $B_{r}=\sum_{p} B_{r, p}$ est $(-1)^{r} \partial, \partial$ désignant l'opérateur bord du complexe des chaînes singulières de $Y \times G^{r}$.

(2) l'opérateur $\partial^{\prime \prime}$, de type $(-1,0)$, dont la restriction à $B_{r}$ est $\sum_{i=0}^{i=r}(-1)^{i} \beta_{r, i}, \beta_{r, i}$ désignant l'homomorphisme de $B_{r}$ dans $B_{r-1}$ défini par l'application $b_{r, i}\left(\partial^{\prime \prime}=0\right.$ sur $\left.B_{0}\right)$.

On a visiblement les relations: $\partial^{\prime 2}=\partial^{\prime \prime 2}=\partial^{\prime} \partial^{\prime \prime}+\partial^{\prime \prime} \partial^{\prime}=0$. On définit donc un nouvel opérateur bord $\Delta$, abaissant le degré total d'une unité, en posant $\Delta=\partial^{\prime}+\partial^{\prime \prime}$. Les groupes d'homologie (resp. les modules de cohomologie) du complexe $B(G, Y)$ ainsi défini seront notés $H_{n}(G, Y)$ (resp. $H^{n}(G, Y)$ ). La filtration de $B(G, Y)$ par les sous-complexes $B_{0}+B_{1}+\cdots+B_{r}$ définit, en homologie, une suite spectrale dont le terme $E^{1}$ est somme directe des groupes d'homologie singulière des espaces $Y \times G^{r}$ (d'une manière précise $E_{r, p}^{1}$ $\left.=H_{p}\left(Y \times G^{r}\right)\right)$. L'opérateur $\partial^{1}$ de $E^{1}$ a pour restriction à $E_{r, p}^{1}$ l'homomorphisme $\sum(-1)^{i}\left(\beta_{r, i}\right)_{*}$, où $\left(\beta_{r, i}\right)_{*}$ est l'homomorphisme de $H_{p}\left(Y \times G^{r}\right)$ dans $H_{p}\left(Y \times G^{r-1}\right)$ défini par l'application $b_{r, i}$.

On précisera maintenant les relations entre la construction précédente et celle qui a fait l'objet du $\S 1$. Soit $R$ la relation d'équivalence dans $Y$ définie par $G$, c'est à dire la relation d'équivalence ayant pour graphe l'ensemble des points $\left(y, y^{\prime}\right) \in Y^{2}$ tels que $y G=y^{\prime} G$. Le quotient de $Y$ par cette relation $R$ sera noté $Y_{G}$. Pour tout entier $r \geqq 0$, on définit une application continue $h_{r}$ de $Y \times G^{r}$ dans $Y_{r}$ en posant

$$
h_{r}\left(y, s_{1}, s_{2}, \cdots, s_{r}\right)=\left(y, y s_{1}, y s_{1} s_{2}, \cdots, y s_{1} s_{2} \cdots s_{r}\right) .
$$

Lorsque $r \geqq 1$ et $r \geqq i \geqq 0$, on a $h_{r-1} b_{r, i}=a_{r, i} h_{r}$. Soit $\eta_{r}$ l'homomorphisme de $B_{r}$ dans $A_{r}$ défini par l'application $h_{r}$. Ces homomorphismes constituent un homomorphisme canonique $\eta$ du complexe bigradué $B(G, Y)$ dans le complexe bigradué $A(R, Y)$. Soient $\eta_{*}: H_{n}(G, Y) \rightarrow H_{n}(R, Y)$ et $\eta^{*}: H^{n}(R, Y)$ $\rightarrow H^{n}(G, Y)$ les homomorphismes canoniques induits par $\eta$. En composant ces homomorphismes avec les homomorphismes $H_{n}(R, Y) \rightarrow H_{n}\left(Y_{G}\right)$ et $H^{n}\left(Y_{G}\right)$ $\rightarrow H^{n}(R, Y)$, on obtient des homomorphismes canoniques que l'on notera $\chi_{*}: H_{n}(G, Y) \rightarrow H_{n}\left(Y_{G}\right)$ et $\chi^{*}: H^{n}\left(Y_{G}\right) \rightarrow H^{n}(G, Y)$.

Soient maintenant $Y$ et $Y^{\prime}$ deux espaces où le groupe $G$ opère de manière continue à droite et soit $t$ une application continue de $Y$ dans $Y^{\prime}$ telle que $t(y s)=t(y) s$ pour tout $y \in Y$ et tout $s \in G$. On notera $t_{G}$ l'application de $Y_{G}$ dans $Y_{G}^{\prime}$ déduite de $t$ par passage aux quotients. Pour tout entier $r$, soit $t_{r}$ l'application continue de $Y \times G^{r}$ dans $Y^{\prime} \times G^{r}$ qui transforme $\left(y, s_{1}, \cdots, s_{r}\right)$ en $\left(t(y), s_{1}, \cdots, s_{r}\right)$. Chaque application $t_{r}$ définit un homomorphisme $\theta_{r}: B_{r}(G, Y) \rightarrow B_{r}\left(G, Y^{\prime}\right)$ et les homomorphismes $\theta_{r}$ constituent un homomorphisme du complexe bigradué $B(G, Y)$ dans le complexe bigradué $B\left(G, Y^{\prime}\right)$. Cet homomorphisme induit des homomorphismes $\theta^{*}: H^{n}\left(G, Y^{\prime}\right)$ $\rightarrow H^{n}(G, Y)$. Si $\theta_{G}^{*}: H^{n}\left(Y_{G}^{\prime}\right) \rightarrow H^{n}\left(Y_{G}\right)$ est l'homomorphisme défini par l'application $t_{G}$, on voit facilement que le diagramme 


$$
\begin{array}{ccc}
H^{n}(G, Y) \stackrel{\theta^{*}}{\longleftarrow} & H^{n}\left(G, Y^{\prime}\right) \\
\uparrow \chi^{*} & \stackrel{\theta_{G}^{*}}{\uparrow} & \uparrow \chi^{*} \\
H^{n}\left(Y_{G}\right) & \stackrel{\longleftarrow}{\longleftarrow} H^{n}\left(Y_{G}^{\prime}\right)
\end{array}
$$

est commutatif.

\section{Cas des espaces fibrés principaux.}

THÉORÈme 2. Si $Y$ est un espace fibré principal de groupe $G$, alors les homomorphismes canoniques $\chi_{*}: H_{n}(G, Y) \rightarrow H_{n}\left(Y_{G}\right)$ et $\chi^{*}: H^{n}\left(Y_{G}\right) \rightarrow H^{n}(G, Y)$ sont bijectifs.

En effet, dans ce cas, les applications $h_{r}: G \times Y^{r} \rightarrow Y_{r}$ sont des homéomorphismes, et par suite les homomorphismes $\eta_{*}$ et $\eta^{*}$ définis au $\$ 2$ sont bijectifs. Le Théorème résulte donc du Théorème 1.

L'isomorphisme du Théorème 2 a été indiqué explicitement dans différents cas, et en particulier des le cas où $G$ est un groupe discret (le complexe $B(G, Y)$ est alors le complexe dans chaînes non homogènes de $G$, au sens de Eilenberg-Mac Lane, à coefficients dans le complexe des chaînes singulières ... de $Y$, cf. $[1 ; 2])$.

Lorsque $Y=I$ est un espace réduit à un point, le complexe $B(G, I)$ ne diffère du complexe obtenu par "bar construction" à partir du complexe $S(G)$ des chaînes singulières de $G$ que dans la mesure où il fait intervenir les complexes des chaînes singulières des espaces $G^{r}$ et non les produits tensoriels $\otimes_{r} S(G)$ (cf. [3]). Lorsque $Y=G$, les opérations de $G$ dans $Y$ étant les translations à gauche, il résulte du Théorème 2 que $B(G, G)$ est "acyclique," ses groupes d'homologie étant isomorphes à ceux de l'espace $G / G$. Ce complexe $B(G, G)$ est, à des détails près, une "construction acyclique" au sens du Séminaire H. Cartan, 1954/55, Exposés 3, 13, 19.

Soit $P$ un espace fibré principal de groupe $G$, de base $P_{G}$. L'application de $P$ sur $I$ (espace réduit à un point) définit des homomorphismes canoniques $H^{n}(G, I) \rightarrow H^{n}(G, P)$ qui, composés avec les isomorphismes $\chi^{*-1}: H^{n}(G, P)$ $\rightarrow H^{n}\left(P_{G}\right)$ donnent des homomorphismes canoniques $\gamma^{*}: H^{n}(G, I) \rightarrow H^{n}\left(P_{G}\right)$. Ces homomorphismes seront appelés les homomorphismes caractéristiques de l'espace fibré $P$.

4. Interprétation des modules $H^{n}(G, Y)$. Soit $\mathfrak{B}$ un espace fibré principal universel de groupe $G$ (c'est à dire un espace fibré principal de groupe $G$ ayant même homologie singulière qu'un point, cf. [7]). Soit d'autre part $F$ un espace où $G$ opère de manière continue à droite. En posant $(u, y) s=(u s, y s)$ pour tout point $(u, y) \in \mathfrak{B} \times F$, on définit dans $\mathfrak{B} \times F$ une structure d'espace fibré principal de groupe $G$ dont la base est un espace fibré universel de fibre $F$ et de groupe $G$. La projection de $\mathfrak{B} \times F$ sur $F$ définit un homomorphisme $\pi$ du complexe bigradué $B(G, \mathfrak{P} \times F)$ dans le complexe bigradué $B(G, F)$. Du fait que $\mathfrak{P}$ a même homologie singulière qu'un point résulte que cet homo- 
morphisme $\pi$ induit un isomorphisme des termes de rang 1 des suites spectrales obtenues en filtrant ces deux complexes par le degré $r$. On en déduit le résultat suivant:

THÉORÈME 3. Si $\mathfrak{B}$ est un espace fibré principal universel de groupe $G$, les homomorphismes canoniques $\pi_{*}: H_{n}(G, \mathfrak{B} \times F) \rightarrow H_{n}(G, F)$ et $\pi^{*}: H^{n}(G, F)$ $\rightarrow H^{n}(G, \mathfrak{B} \times F)$ sont bijectifs.

Corollaire. Si $\leftleftarrows$ est un espace fibré universel de fibre $F$ et de groupe $G, i l$ existe pour tout entier $n$ un isomorphisme canonique de $H^{n}(G, F)$ sur $H^{n}(\mathbb{E})$.

On l'obtient en composant $\pi^{*}$ avec l'isomorphisme $\chi^{*-1}: H^{n}(G, \mathfrak{B} \times F)$ $\rightarrow H^{n}(\mathbb{F})$ (cf. Théorème 2). En particulier, les modules $H^{n}(G, I)$ sont canoniquement isomorphes aux modules de cohomologie de l'espace classifiant $P_{G}$.

Soit maintenant $P$ un espace fibré principal quelconque de groupe $G$, et soit $r$ une application continue de $P$ dans $F$ telle que $r(y s)=r(y) s$ quels que soient $y \in P$ et $s \in G$. Une telle application correspond à une section continue $\uparrow_{G}$ de l'espace fibré de fibre $F$ associé à $P$ qui s'obtient par passage aux quotients à partir de l'homomorphisme $p: y \rightarrow(y, r(y))$ de $P$ dans $P \times F$. L'application $r$ définit des homomorphismes $\rho^{*}: H^{n}(G, F) \rightarrow H^{n}(G, P)$. On va interpréter les homomorphismes $\chi^{*-1} \rho^{*}: H^{n}(G, F) \rightarrow H^{n}\left(P_{G}\right)$ dans le cas où il existe un homomorphisme $t$ de $P$ dans un espace fibré universel $\mathfrak{P}$ de groupe $G\left({ }^{3}\right)$. Désignons encore par $t$ l'application de $P \times F$ dans $\Re \times F$ qui prolonge $t$ et notons $p$ la projection de $\mathfrak{B} \times F$ sur $F$ : on a alors $r=p t \hat{r}$. D'autre part, on a le diagramme commutatif:

$$
\begin{gathered}
H^{n}(G, P) \stackrel{\hat{\rho}^{*}}{\stackrel{1}{\leftrightarrows}} H^{n}(G, P \times F) \stackrel{\theta^{*}}{\longleftarrow} H^{n}(G, P \times F) \stackrel{\pi^{*}}{\longleftarrow} H^{n}(G, F) \\
\uparrow \chi^{*} \quad \stackrel{\hat{\rho}_{G}^{*}}{\longleftarrow} H^{n}\left((P \times F)_{G}\right) \stackrel{\theta_{G}^{*}}{\longleftarrow} H^{n}\left((P \times F)_{G}\right)
\end{gathered}
$$

et par suite: $\chi^{*-1} \rho^{*}=\chi^{*-1} \hat{\rho}^{*} \theta^{*} \pi^{*}=\hat{\rho}_{G}^{*} \theta_{G}^{*} \chi^{*-1} \pi^{*}$. On voit donc que $\chi^{*-1} \rho^{*}$ est composé: (1) de l'isomorphisme canonique de $H^{n}(G, F)$ sur le $n$-ième module de cohomologie de l'espace fibré universel $(\mathfrak{B} \times F)_{G}$, (2) de l'homomorphisme $\theta_{G}$ défini par l'application $t_{G}$ de l'espace fibré $(P \times F)_{G}$ dans l'espace fibré universel $(\mathfrak{B} \times F)_{G}$, (3) de l'homomorphisme $\hat{\rho}_{G}^{*}$ défini par la section de l'espace fibré $(P \times F)_{G}$.

En appliquant ce qui précède au cas $F=I$, on obtient ce qui suit:

THÉORÈme 4. Si il existe un homomorphisme $t$ de l'espace fibré principal $P$ dans un espace fibré principal universel $\mathfrak{B}$, alors l'homomorphisme caractéristique $H^{n}(G, I) \rightarrow H^{n}\left(P_{G}\right)$ est composé de l'isomorphisme canonique de $H^{n}(G, I)$ sur $H^{n}\left(\mathfrak{B}_{G}\right)$ et de l'homomorphisme de $H^{n}\left(\mathfrak{B}_{G}\right)$ dans $H^{n}\left(P_{G}\right)$ que définit l'application déduite de $t$ par passage aux quotients.

(') On sait qu'un tel homomorphisme $t$ existe lorsque la base $P_{G}$ de $\mathrm{P}$ est paracompacte et localement homéomorphe à un complexe simplicial fini. 
L'image de l'homomorphisme caractéristique est donc constituée dans ce cas par les classes caractéristiques au sens habituel du terme.

5. Multiplicateurs et espaces fibrés. Dans ce paragraphe, on désigne par $R$ une relation d'équivalence dans un espace topologique $Y$ et par $q$ l'application canonique de $Y$ sur l'espace quotient $X=Y / R$.

Soit $P$ un espace fibré principal de base $X$, de groupe $G$, trivialisé par l'application $q: Y \rightarrow X$ et soit $p$ la projection de $P$ sur $X$. Il existe une application continue $r: Y \rightarrow P$ telle que $q=p r$. Soit $f$ l'application du graphe $Y_{1}$ dans $G$ définie par la condition $r\left(y^{\prime}\right)=r(y) f\left(y, y^{\prime}\right)$ pour tout point $\left(y, y^{\prime}\right) \in Y_{1}$. Cette application $f$ vérifie la condition:

(M) $f$ est continue et lorsque $y \sim y^{\prime} \sim y^{\prime \prime}$, on a

$$
f\left(y, y^{\prime \prime}\right)=f\left(y, y^{\prime}\right) f\left(y^{\prime}, y^{\prime \prime}\right) .
$$

De la trivialité locale de $P$ résulte que $f$ vérifie même la condition plus forte:

$\left(\mathrm{M}^{\prime}\right)$ pour tout point $x \in X$, il existe un voisinage ouvert $U$ de $x$ dans $X$ et une application continue $g$ de $q^{-1}(U)$ dans $G$ telle que $f\left(y, y^{\prime}\right)=g^{-1}(y) g\left(y^{\prime}\right)$ pour tout point $\left(y, y^{\prime}\right) \in Y$ tel que $q(y) \in U$.

Soit $r^{\prime}$ une autre application continue de $Y$ dans $P$ telle que $q=p r^{\prime}$ et soit $f^{\prime}$ l'application de $Y_{1}$ dans $G$ définie par la condition $r^{\prime}\left(y^{\prime}\right)=r^{\prime}(y) f^{\prime}\left(y, y^{\prime}\right)$ pour $\left(y, y^{\prime}\right) \in Y_{1}$. Il existe une application continue $g$ de $Y$ dans $G$ telle que $r^{\prime}(y)=r(y) g(y)$ pour tout $y \in Y$. Par conséquent, $f$ et $f^{\prime}$ vérifient la condition:

(E) il existe une application continue $g$ de $Y$ dans $G$ telle que

$$
f^{\prime}\left(y, y^{\prime}\right)=g^{-1}(y) f\left(y, y^{\prime}\right) g\left(y^{\prime}\right)
$$

pour tout point $\left(y, y^{\prime}\right) \in Y_{1}$.

Toute application $f$ de $Y_{1}$ dans $G$ qui vérifie la condition $\left(\mathrm{M}^{\prime}\right)$ sera appelée un multiplicateur (sur $Y$ à valeurs dans $G$ ). Deux multiplicateurs $f$ et $f^{\prime}$ qui vérifient la condition (E) seront dits équivalents. L'ensemble des classes de multiplicateurs équivalents pour cette relation (E) sea noté $M(Y, G)$. On observera que $\left(\mathrm{M}^{\prime}\right)$ est conséquence de $(\mathrm{M})$ toutes les fois que, pour tout point $x_{0} \in X$, il existe un voisinage ouvert $U$ de $x_{0}$ et une application continue $t$ de $U$ dans $Y$ telle que $q(t(x))=x$ pour tout $x \in U$.

Ce qui précède montre comment, à tout espace fibré principal de base $X$ et de groupe $G$ trivialisé par $q$ est associé une classe de multiplicateurs $\in M(Y, G)$. A deux espaces fibrés isomorphes correspond évidemment la même classe de multiplicateurs. On a donc une application naturelle $\Psi$ de l'ensemble des classes de fibrés principaux de base $X$ de groupe $G$ trivialisés par $q$ dans l'ensemble $M(Y, G)$. Cette application $\Psi$ est bijective. Pour le voir, on va montrer que tout multiplicateur $f$ sur $Y$ à valeurs dans $G$ définit canoniquement un espace fibré principal de base $X$, de groupe $G$ trivialisé par $q$. Dans l'espace produit $Y \times G$, on définit une relation d'équivalence en posant $(y, s) \sim\left(y^{\prime}, s^{\prime}\right)$ lorsque $y \sim y^{\prime}$ et $s=f\left(y, y^{\prime}\right) s^{\prime}$. Soit $P$ l'espace topo- 
logique quotient de $Y \times G$ par cette relation d'équivalence et soit $h$ l'applica tion canonique de $Y \times G$ sur $P$. L'application $(y, s) \rightarrow q(y)$ de $Y \times G$ sur $X$ est composée de $h$ et d'une application continue $p$ de $P$ sur $X$. D'autre part, il existe une loi d'opération de $G$ dans $P$ et une seule telle que $h(y, s) t=h(y, s t)$ pour $y \in Y$ et $s, t \in G$. On vérifie que $P$, muni de ces opérations de $G$ et de l'application $p$ est un espace fibré principal de base $X$ et de groupe $G$. Il est trivialisé par $q$ car $q(y)=p(h(y, e))$ (e=élément neutre de $G)$. Puisque $h\left(y^{\prime}, e\right)=h\left(y, f\left(y, y^{\prime}\right)\right)=h(y, e) f\left(y, y^{\prime}\right)$, la classe de multiplicateur associée à $P$ est la classe de $f$. Ceci prouve que $\Psi$ est surjective. Soient maintenant $P^{\prime}$ un espace fibré principal de base $X$, de groupe $G$ trivialisé par $q$ et $g^{\prime}$ une application de $Y$ dans $P^{\prime}$ telle que $q=p^{\prime} g^{\prime}$. Si le multiplicateur $f^{\prime}$ défini par $g^{\prime}\left(y^{\prime}\right)=g^{\prime}(y) f^{\prime}\left(y, y^{\prime}\right)$ est équivalent à $f$, il existe une application continue $g^{\prime \prime}$ de $Y$ dans $P^{\prime}$ telle que $q=p^{\prime} g^{\prime \prime}$ et $g^{\prime \prime}\left(y^{\prime}\right)=g^{\prime \prime}(y) f\left(y, y^{\prime}\right)$ lorsque $y \sim y^{\prime}$. Soit $b$ l'application continue de $P$ dans $P^{\prime}$ définie par la condition $b(h(y, s))=g^{\prime \prime}(y) s$ pour tout $y \in Y$ et tout $s \in G$. On a $p=p^{\prime} b$ et $b(z s)=b(z) s$ pour tout $z \in P$ et tout $s \in G$. Par conséquent $b$ est un isomorphisme de $P$ sur $P^{\prime}$ et ceci prouve que $\Psi$ est injective $\left({ }^{4}\right)$.

Lorsque $Y$ et $R$ sont construits à partir d'un recouvrement ouvert de $X$, comme dans l'exemple donné ou paragraphe 1 , on voit que les multiplicateurs sur $Y$ à valeurs dans $G$ ne sont pas autre chose que les systèmes de fonctions de passage à valeurs dans $G$ pour le recouvrement donné. Lorsque $Y$ est un espace fibré principal de groupe $\Gamma$ et que $R$ est le relation d'équivalence définie par les opérations de $\Gamma$, alors, en identifiant $Y_{1}$ et $Y \times \Gamma$ par l'homéomorphisme $(y, s) \rightarrow(y, y s)$, on voit que les multiplicateurs sur $Y$ à valeurs dans $G$ sont les application continues $k: Y \times \Gamma \rightarrow G$ telles que $k(y, s t)$ $=k(y, s) k(y s, t)(y \in Y, s, t \in \Gamma)$. Les multiplicateurs sont donc dans ce cas les "facteurs d'automorphie."

6. Homomorphismes caractéristiques et multiplicateurs. Soit $R$ une relation d'équivalence dans l'espace topologique $Y$ et soit $X$ l'espace quotient $Y / R$. Soit $f$ un multiplicateur sur $Y$ à valeurs dans un groupe topologique $G$. Le multiplicateur $f$ définit un espace fibré principal $P$ de groupe $G$ et de base $X$. On va donner une construction de l'homomorphisme caractéristique $H^{n}(G, I) \rightarrow H^{n}(X)$ de ce fibré, utilisant le complexe $A(R, Y)$ et le multiplicateur $f$.

Soit $F$ un espace topologique où le groupe $G$ opère à droite de manière continue et soit $c$ une application continue de $Y$ dans $F$ telle que $c\left(y^{\prime}\right)$ $=c(y) f\left(y, y^{\prime}\right.$ ) lorsque $y \sim y^{\prime}$ (une telle application correspond à une section continue de l'espace fibré de fibre $F$ associé à $P$ ). On va montrer que le

(4) Le groupe des applications continues de $Y$ dans $G$ opère dans l'ensemble des multiplicateurs sur $Y$ à valeurs dans $G$, le transformé d'un multiplicateur $f$ par une application $g$ étant le multiplicateur $(f g)\left(y, y^{\prime}\right)=g(y)^{-1} f\left(y, y^{\prime}\right) g\left(y^{\prime}\right)$. On vérifie facilement que le groupe de stabilité d'un multiplicateur $f$ est canoniquernent isomorphe au groupe des automorphismes de l'espace. fibré principal défini par $f$. 
multiplicateur $f$ et l'application $c$ définissent un homomorphisme du complexe bigradué $A(R, Y)$ dans le complexe bigradué $B(G, F)$. Pour tout entier $r \geqq 0$, on notera $(c, f)_{r}$ l'application continue de $Y_{r}$ dans $F \times G^{r}$ définie par

$$
(c, f)_{r}\left(y_{0}, y_{1}, \cdots, y_{r}\right)=\left(c\left(y_{0}\right), f\left(y_{0}, y_{1}\right), f\left(y_{1}, y_{2}\right), \cdots, f\left(y_{r-1}, y_{r}\right)\right)
$$

pour tout $\left(y_{0}, y_{1}, \cdots, y_{r}\right) \in Y_{r}$. Lorsque $0 \leqq i \leqq r$, on a $b_{r, i}(c, f)_{r}=(c, f)_{r-1} a_{r, i}$. De ces relations résulte que les applications $(c, f)_{r}$ définissent des homomorphismes $(\gamma, \phi)_{r}: A_{r}(R, Y) \rightarrow B_{r}(G, F)$ qui constituent un homomorphisme $(\gamma, \phi)$ du complexe bigradué $A(R, Y)$ dans le complexe bigradué $B(G, F)$.

Soit $g$ une application continue de $Y$ dans $G$. Le multiplicateur $f^{\prime}\left(y, y^{\prime}\right)$ $=g(y)^{-1} f\left(y, y^{\prime}\right) g\left(y^{\prime}\right)$ et l'application $c^{\prime}(y)=c(y) g(y)$ définissent un nouvel homomorphisme $\left(\gamma^{\prime}, \phi^{\prime}\right)$ de $A(R, Y)$ dans $B(G, Y)$. Le lemme suivant montre que $\left(\gamma^{\prime}, \phi^{\prime}\right)$ et $(\gamma, \phi)$ sont homotopes:

Lemme. Il existe un homomorphisme $\eta d u$ groupe $A(R, Y)$ dans le groupe $B(G, F)$ tel que:

$$
\begin{aligned}
\eta\left(A_{r, p}\right) & \subset B_{r+1, p}, \\
\eta \partial^{\prime}+\partial^{\prime} \eta & =0, \\
\eta \partial^{\prime \prime}+\partial^{\prime \prime} \eta & =\left(\gamma^{\prime}, \phi^{\prime}\right)-(\gamma, \phi) .
\end{aligned}
$$

Pour tout couple d'entiers $(r, i)$ tels que $r \geqq i \geqq 0$, désignons en effet par $h_{r, i}$ l'application continue de $Y_{r}$ dans $F \times G^{r+1}$ définie par:

$$
\begin{aligned}
& h_{r, i}\left(y_{0}, y_{1}, \cdots, y_{r}\right) \\
& \quad=\left(c\left(y_{0}\right), f\left(y_{0}, y_{1}\right), \cdots, f\left(y_{i-1}, y_{i}\right), g\left(y_{i}\right), f^{\prime}\left(y_{i}, y_{i+1}\right), \cdots, f^{\prime}\left(y_{r-1}, y_{r}\right)\right) .
\end{aligned}
$$

Soit $\eta_{r, i}$ l'homomorphisme de $A_{r}(R, Y)$ dans $B_{r}(G, F)$ défini par l'application $h_{r, i}$ et soit $\eta$ l'homomorphisme du groupe $A(R, Y)$ dans le groupe $B(G, F)$ ayant $\sum_{i=0}^{i=r}(-1)^{i} \eta_{r, i}$ pour restriction à $A_{r}(R, Y)$. On vérifie directement que $\eta$ satisfait aux conditions du lemme.

En appliquant ce qui précède au cas $F=I$, on voit que toute classe de multiplicateurs sur $Y$ à valeurs dans $G$ définit pour tout entier $n$ un homomorphisme $H^{n}(G, I) \rightarrow H^{n}(R, Y)$.

THÉORÈmE 5. Soit $f$ un multiplicateur sur $Y$ d valeurs dans $G$ et soit $P$ l'espace fibré principal de base $Y / R$ de groupe $G$ défini par f; l'homomorphisme $\phi^{*}: H^{n}(G, I) \rightarrow H^{n}(R, Y)$ défini par $f$ est composé de l'homomorphisme caractéristique $H^{n}(G, I) \rightarrow H^{n}(Y / R)$ de l'espace fibré $P$ et de l'homomorphisme canonique $\chi^{*}: H^{n}(Y / R) \rightarrow H^{n}(R, Y)$.

Soit en effet $g$ une application de $Y$ dans $P$ telle que $g\left(y^{\prime}\right)=g(y) f\left(y, y^{\prime}\right)$ lorsque $y \sim y^{\prime}$ et soit $p$ la projection de $P$ sur $Y / R$. Le multiplicateur $f$ et l'application $g$ définissent un homomorphisme $(\gamma, \phi)$ du complexe $A(R, Y)$ 
dans le complexe $B(G, P)$. En composant $(\gamma, \phi)$ avec l'homomorphisme $\theta: B(G, P) \rightarrow B(G, I)$ que définit l'application $P \rightarrow I$, on obtient l'homomorphisme de $A(R, Y)$ dans $B(G, I)$ défini par $f$. D'autre part, puisque $q=p g$, le diagramme

$$
H^{n}(R, \underset{\nwarrow}{Y}) \underset{\chi_{n}^{*}(Y / R)}{\stackrel{(\gamma, \phi)^{*}}{\pi^{*}}} H^{n}(G, P)
$$

est commutatif ( $\pi^{*}$ étant l'isomorphisme canonique du Théorème 2 ). On a donc $\phi^{*}=(\gamma, \phi)^{*} \theta^{*}=\chi^{*} \pi^{*-1} \theta^{*}$, ce qui démontre le théorème. Lorsque $H^{n}(X) \rightarrow H^{n}(R, Y)$ est bijectif, l'homomorphisme $\phi^{*}$ permet donc d'obtenir les classes caractéristiques de l'espace fibré $P$.

Indiquons pour terminer comment cette méthode s'applique au cas des espaces fibrés principaux de groupe $C^{*}=C-(0)$ (cf. [5]).

Désignons par $c$ le cocycle de degré 1 de l'espace $C$ défini par $c(\tau)=(1 / 2 \pi i)$ $\cdot \int_{\tau} d z / z$ pour tout simplexe singulier $\tau$ de dimension 1 dans $C^{*}$. Ce cocycle peut être considéré comme une cochaîne de degré 2 du complexe $\operatorname{Hom}_{Z}\left(B\left(C^{*}, I\right), C\right)$. On a $d^{\prime}(c)=c \partial^{\prime}=0$ et $d^{\prime \prime}(c)=c \partial^{\prime \prime}=0$, donc $c$ est un cocycle de ce complexe. La classe de Chern (pour des coefficients dans $C$ ) d'un espace fibré principal de groupe $C^{*}$ est l'image de la classe de $c$ dans $H^{2}\left(C^{*}, I\right)$ par l'homomorphisme caractéristique.

Soit $Y$ un espace fibré principal de base $X$ et de groupe $\Gamma$ discret. Soit $f$ un multiplicateur sur $Y$ à valeurs dans $C^{*}$, qu'on écrira comme application de $Y \times \Gamma$ dans $C^{*}$. La classe de Chern de l'espace fibré principal $P_{f}$ défini par $f$ a pour image dans $H^{2}(\Gamma, Y)$ la classe du cocycle $\hat{c}_{1}$, image réciproque de $c_{1}$ par l'application $f: Y \times \Gamma \rightarrow C^{*}$. Supposons que $Y$ soit simplement connexe; il existe alors une application continue $g: Y \times \Gamma \rightarrow C$ telle que $f(y, s)=e^{2 \pi i g(y, s)}$. On peut considérer $g$ comme une 0 -cochaîne de l'espace $Y \times \Gamma$, donc comme une 1-cochaîne du complexe $\operatorname{Hom}_{z}(B(\Gamma, Y), C)$. On a $\hat{c}_{1}=-d^{\prime}(g)$, donc $\hat{c}_{1}$ est cohomologue à $d^{\prime \prime}(g)$. Or $d^{\prime \prime}(g)$ est la 0 -cochaîne de l'espace $Y \times \Gamma \times \Gamma$ définie par $\left(d^{\prime \prime}(g)\right)(y, s, t)=g(y s, t)-g(y, s t)+g(y, s)=e(s, t)$; elle est à valeurs entières et indépendante de $y$. On voit donc que la classe de Chern (à coefficients dans $C$ ) de l'espace fubré $P_{f}$ est l'image par l'homomorphisme caractêristique $H^{2}(\Gamma, I) \rightarrow H^{2}(X)$ de la classe du 2-cocycle $e(s, t)$. Ce résultat est encore valable pour des coefficients entiers. La classe de Chern à coefficients entiers s'obtiendra en partant d'un cocycle de $\operatorname{Hom}_{Z}\left(B\left(C^{*}, I\right), Z\right)$ de la forme $c_{1}+\Delta b$, où $b$ est une 0 -cochaîne sur l'espace $C^{*}$, à valeurs dans $C$, telle que $z=e^{2 \pi i b(z)}$ pour tout $z \in C$. La classe de Chern de $P_{f}$ a pour image dans $H^{2}(\Gamma, Y)$ la classe du cocycle $\hat{c}+\Delta \hat{b}$, où $\hat{b}$ est l'image réciproque de $b$ par l'application $f$; ce cocycle est encore cohomologue à $e$ dans le complexe de cochaînes $\operatorname{Hom}_{Z}(B(\Gamma, Y), Z)$. 


\section{Bibliographie}

1. H. Cartan and S. Eilenberg, Homological algebra, Princeton, 1956.

2. H. Cartan and J. Leray, Relations entre anneau d'homologie et groupe de Poincarê, Colloque de Topologie algébrique, C.N.R.S., Paris, 1949, pp. 83-85.

3. S. Eilenberg and S. MacLane, On the groups $H(\pi, n)$, I, Ann. of Math. vol. 58 (1953) pp. 55-106.

4. S. Eilenberg and N. Steenrod, Foundations of algebraic topology, Princeton, 1952.

5. R. C. Gunning, The structure of factors of automorphy, Amer. J. Math. vol. 78 (1956) pp. 357-382.

6. J. Leray, Sur la forme des espaces topologiques et sur les points fixes des représentations, J. Math. Pures Appl. vol. 24 (1945) pp. 169-199. 436.

7. J. Milnor, Construction of universal bundles, II, Ann. of Math. vol. 63 (1956) pp. 430-

UNIVERSITÉ DE STRASBOURG,

Strasbourg, France 